\section{Risk of severe infection following rituximab and the efficacy of antimicrobial prophylaxis}

We read with interest the article 'Trimethoprim-sulfamethoxazole prophylaxis prevents severe/life-threatening infections following rituximab in antineutrophil cytoplasm antibodyassociated vasculitis' by Kronbichler et al. ${ }^{1}$ Severe infections continue to be a significant cause of morbidity and mortality in patients with anti-neutrophil cytoplasmic antibody-associated vasculitis (AAV), and we commend the authors for pursuing this study. However, we have several concerns regarding their methodologies.

First, more than $90 \%$ of patients with AAV analysed were prevalent cases. This may limit the generalisability of their findings to incident cases in whom there has been no prior treatment with cyclophosphamide or other immunosuppressive agents. As the authors note, there was an average delay of 4 years between initial diagnosis and rituximab initiation, during which time the patients received a variety of immunosuppressive medications, including large quantities of glucocorticoids. These treatments likely contributed to the risk of infection observed by the authors.

Second, it is unclear whether the authors accounted for the fact that exposure to trimethoprim-sulfamethoxazole was timevarying. In other words, were time and outcomes (eg, infections) after antibiotic discontinuation attributed to the unexposed arm (eg, no antibiotic)? If antibiotic prophylaxis was discontinued during follow-up, subsequent infections should have been attributed to the unexposed arm.

Third, the authors did not account for confounding by indication or contraindication. They identified certain patients, particularly those with head and neck disease, as those more likely to receive prophylaxis. This greater likelihood of prophylaxis stems, no doubt, from the belief by some investigators that therapy with trimethoprim-sulfamethoxazole diminishes the risk of disease flares in the upper respiratory tract. ${ }^{2}$ Moreover, patients with head and neck disease may be more likely to have 'limited' disease and less likely to have disease that is 'disseminated'. ${ }^{3}$ Such patients are less likely to receive intensive immunosuppressive therapy with drugs such as cyclophosphamide or high-dose glucocorticoids for prolonged periods of time and inherently less likely to suffer from infectious complications of treatment. Answering the question evaluated by the authors therefore requires an approach to account for this potential confounding.

Finally, the potential adverse effects associated with sulfa medications should not be minimised. In the trial by Stegeman et $a l^{2}{ }^{2} 20 \%$ of patients had to discontinue trimethoprimsulfamethoxazole because of side effects. Life-threatening hypersensitivity reactions including Stevens-Johnson syndrome, interstitial nephritis, thrombocytopaenia, liver function test abnormalities and drug-resistant infections can all occur with chronic antimicrobial prophylaxis. ${ }^{4}$ The risks of adverse effects for patients receiving immunosuppressive treatment for AAV are not uniform and likely lower than in earlier eras, when cyclophosphamide and high-dose glucocorticoid regimens dominated the approaches to treatment.
Further studies are necessary to quantify these risks in contemporary cohorts.

Although we also believe that trimethoprim-sulfamethoxazole prophylaxis has a role in the prevention of infectious adverse events in AAV, the full range of risks as well as potential benefits need to be more clearly understood.

\section{Zachary Scott Wallace $\odot{ }^{1,2,3}$ Hyon Choi, ${ }^{1,2,3}$ John H Stone ${ }^{1,3}$}

${ }^{1}$ Rheumatology Unit, Division of Rheumatology, Allergy and Immunology, Massachusetts General Hospital, Boston, USA

${ }^{2}$ Clinical Epidemiology Unit, Division of Rheumatology, Allergy and Immunology, Harvard Medical School, Boston, USA

${ }^{3}$ Massachusetts General Hospital and Harvard Medical School, Boston, USA

Correspondence to Dr Zachary Scott Wallace, Rheumatology Unit, Clinical Epidemiology Unit, Division of Rheumatology, Allergy, and Immunology, Massachusetts General Hospital, Harvard Medical School, Boston, MA 02114, USA zswallace@mgh.harvard.edu

Handling editor Josef S Smolen

Contributors All named authors contributed to planning, conduct and reporting of the work.

Funding ZSW received grant support through a Scientist Development Award from the Rheumatology Research Foundation and from the National Institute of Arthritis and Musculoskeletal and Skin Diseases (NIAMS/NIH; Loan Repayment Award and K23 AR073334).

Competing interests None declared.

Patient consent Not required.

Provenance and peer review Not commissioned; internally peer reviewed. Data sharing statement There are no novel data reported here.

(c) Author(s) (or their employer(s)) 2020. No commercial re-use. See rights and permissions. Published by BMJ.

\section{Check for updates}

To cite Wallace ZS, Choi H, Stone JH. Ann Rheum Dis 2020;79:e40.

Received 1 January 2019

Accepted 5 January 2019

Published Online First 30 January 2019

\section{S Linked}

- https://doi.org/10.1136/annrheumdis-2019-215036

Ann Rheum Dis 2020;79:e40. doi:10.1136/annrheumdis-2019-215009

\section{ORCID iD}

Zachary Scott Wallace http://orcid.org/0000-0003-4708-7038

\section{REFERENCES}

1 Kronbichler A, Kerschbaum J, Gopaluni S. Trimethoprim-sulfamethoxazole prophylaxis prevents severe/life-threatening infections following rituximab in antineutrophil cytoplasm antibody-associated vasculitis. Ann Rheum Dis 2018:30(Suppl 1).

2 Stegeman CA, Tervaert JW, de Jong PE, et al. Trimethoprim-sulfamethoxazole (co-trimoxazole) for the prevention of relapses of Wegener's granulomatosis. Dutch Co-Trimoxazole Wegener Study Group. N Eng/ J Med 1996;335:16-20.

3 De Groot K, Rasmussen N, Bacon PA, et al. Randomized trial of cyclophosphamide versus methotrexate for induction of remission in early systemic antineutrophil cytoplasmic antibody-associated vasculitis. Arthritis Rheum 2005;52:2461-9.

4 Fox BC, Sollinger HW, Belzer FO, et al. A prospective, randomized, double-blind study of trimethoprim-sulfamethoxazole for prophylaxis of infection in renal transplantation: Clinical efficacy, absorption of trimethoprim-sulfamethoxazole, effects on the microflora, and the cost-benefit of prophylaxis. Am J Med 1990;89:255-74. 